Entschuldigungen haben in der Medizin einen schlechten Ruf. Dabei zeigen aufrichtige Worte des Bedauerns, dass der Arzt aufmerksam, empathisch und anspruchsvoll ist. Das verbessert nachweislich die Gesprächsatmosphäre. Haftungsrechtliche Folgen gibt es nicht.

Wer sich verteidigt, klagt sich an - so sagt es das Sprichwort. Und wer sich entschuldigt, trägt Schuld - so vermuten es zumindest immer noch viele Mediziner. Entschuldigungen in der Medizin erfreuen sich in Studien wachsender Beliebtheit; in der Praxis allerdings scheuen sich die meisten Ärztinnen und Ärzte ${ }^{1}$, Entschuldigungen auszusprechen. Schließlich haben sie in der Regel nichts falsch gemacht. Und falls doch, so wollen sie keine schlafenden Hunde wecken.

\section{Früher konnten}

\section{Entschuldigungen als Schuldeingeständnis gewertet werden}

Jahrzehntelang hatte die Praxis des Schweigens einen guten Grund: Entschuldigungen konnten als Schuldeingeständnis für mögliche Behandlungsfehler gewertet werden - mit dramatischen Folgen. Bei einer „Schuldanerkenntnis“ von Ärztin oder Arzt erlosch der Anspruch auf Versicherungsleistungen der ärztlichen Haftpflichtversicherung. Und weil nirgends definiert war, was genau eine Schuldanerkenntnis war, verzichteten Mediziner lieber grundsätzlich auf jede bedauernde, empathische, konziliante oder auch nur annähernd entschuldigende Äußerung und machten damit menschlich und medizinisch einen schweren Fehler.

1 Im Folgenden verwendet dieser Text aus Gründen der Lesbarkeit das generische Maskulinum. „Arzt“ meint ärztlich tätige Person, „Patient“ eine Person, die in ärztlicher Behandlung ist - unabhängig von ihrem biologischen Geschlecht.

\section{8 änderte der \\ Gesetzgeber das \\ Versicherungsvertragsgesetz}

Deshalb änderte der Gesetzgeber 2008 das Versicherungsvertragsgesetz. Vertragsklauseln, die die Versicherungen wegen einer Schuldanerkenntnis von der Leistungspflicht befreien, sind hinfällig - selbst wenn sie in Ihrem Vertrag stehen, so haben sie keine Gültigkeit.

Es ist also nicht nur gefahrlos, es ist auch geboten, bei jeder passenden Gelegenheit den Patienten ein Wort des Bedauerns zu sagen.

In den USA sorgen sogenannte „Apology Laws“ dafür, dass ärztliche Entschuldigungen keine juristischen Folgen haben. Seit ihrer Einführung in 36 Bundesstaaten einigte man sich in Arzthaftungsprozessen schneller, und die Entschädigungszahlungen sanken dramatisch - wobei nicht einmal untersucht ist, in welchem Maße die Ärzte die neue Freiheit nutzen „I am sorry“ zu sagen.

\section{Entschuldigung:}

\section{souveränes Mittel zur Entschärfung der Lage}

Der Klinikbetrieb bietet in der Regel reichlich Gelegenheit und Anlass, Umstände und Abläufe, Unannehmlichkeiten und Ärgernisse zu bedauern, auch ohne dass etwas wirklich falsch läuft oder jemandem ein ernsthafter medizinischer Fehler unterläuft. In den seltensten Fällen führen Behandlungsfehler zu Unbehagen; in der Regel beklagen sie den ganz normalen Klinikalltag, Schicksal, Zufall und Pech. Missverständliche Anweisungen, eilige und ungenügende Aufklärung, Wartezeiten, unvollständige Unterlagen, fehlende Arztbriefe, besetzte Telefonleitungen, mühselige Wege, Parkplatzprobleme, fehlendes Faxpapier, verlorene Labordaten, unbequeme Warte- situationen, Verzögerungen beim Röntgen, ungeschicktes Blutabnehmen sind alltäglich. Dazu kommen Ängste, Schmerzen, Nebenwirkungen, Ärger und Langeweile, Scham und Verständnisprobleme - und damit allzu oft eine Gemengelage, die Patienten unzufrieden und dünnhäutig macht. Das sind keine guten Voraussetzungen für ein konstruktives Arztgespräch.

Wenn Sie die emotionale Situation des Patienten mit einer Entschuldigung ansprechen, können Sie die Lage schnell entschärfen - und das sollten Sie tun, auch wenn Sie persönlich keinerlei Verantwortung für die missliche Lage des Patienten und seinen begründeten oder unbegründeten Ärger tragen. „Sie haben sich 2 Tage lang Sorgen gemacht, weil wir Sie nicht angerufen haben? Das tut mir sehr leid. Das war ein Missverständnis. Wenn nichts Auffälliges ist, warten wir einfach bis zum nächsten Termin“. Oder: „Ich sehe mit Schrecken, dass Sie schon seit 11:00 Uhr hier warten. Das tut mir leid. Wir mussten einen Notfall dazwischenschieben. Vielen Dank für Ihre Geduld“.

Wer sich entschuldigt, klagt sich nicht an, sondern zeigt, dass er wahrgenommen hat, dass etwas nicht optimal gelaufen ist. Tatsächlich ist eine Entschuldigung ein Zeichen von Souveränität, sie wirkt empathisch, aufmerksam, vertrauensbildend und einfühlsam. Nach dem Motto: „Ich sehe, dass etwas für Sie unangenehm ist und ich habe den Anspruch, dass es besser läuft. Ich bedaure, dass es Nebenwirkungen, Wartezeiten, Schmerzen, Unstimmigkeiten gibt, weil ich als Arzt nicht zufrieden bin, wenn der Patient es nicht auch ist“.

\section{Entschuldigungen haben einen messbaren medizinischen Effekt}

Dass aufrichtige Entschuldigungen sogar einen messbaren medizinischen Effekt haben, zeigt eine Studie der University of 
Massachusetts [1]. Die Mediziner ließen 184 Versuchspersonen Rechenaufgaben lösen, während sie gestört und belästigt wurden. Erwartungsgemäß stiegen der Kortisonspiegel im Speichel, der Blutdruck und die Herzfrequenz an. Eine ehrliche Entschuldigung ließ die Stresssymptome schnell verschwinden. Nur eine halbherzig dahingeworfene Entschuldigung hatte keinen physischen Effekt.

Dass selbst vollkommen unsinnige und überflüssige Entschuldigungen das Verhalten positiv beeinflussen, zeigt ein verblüffendes Experiment der Harvard Business School [2]. Die Forscher ließen einen Schauspieler bei Nieselregen Passanten ansprechen, um für einen Anruf das Handy auszuleihen. Eröffnete er mit „Tut mir leid, dass es regnet “, waren $47 \%$ der angesprochenen bereit, ihr Handy zu verleihen. Ohne ein „Tut mir leid“ zur Eröffnung gaben nur $9 \%$ der Passanten ihr Telefon her. Ganz offensichtlich wirkt die Entschuldigung als Türöffner, sie schafft eine Gesprächsatmosphäre, die es Menschen leichter macht, freundlich zu sein und kooperativ.

Die Tatsache, dass wohl niemand auf die Idee käme, den Schauspieler ernsthaft für den Regen verantwortlich zu machen, zeigt, dass Menschen gar nicht dazu neigen, Entschuldigung und Schuld oder Ursache zusammenzubringen. Die Formulierung „Es tut mir leid“ schafft unabhängig vom Verursacherprinzip eine entspannte, offene Gesprächsebene, vielleicht weil der junge Mann damit zeigt, dass er zur Kenntnis genommen hat, dass der Passant im Regen steht und dass das unschön ist. Das zeugt von Aufmerksamkeit und ist damit sympathisch. Es ist also nicht wahrscheinlich, dass dem Arzt, der lästige Wartezeiten bedauert, die Schuld am schlechten Zeitmanagement der Klinik gegeben wird.

Werden Missstände (auch vermeintlich banale) nicht angesprochen, so stellt sich bei vielen Patienten ein ungutes Gefühl ein, weil sie vermuten, dass es der Klinik und den Ärzten entweder egal sei, dass der Patient leidet oder aber - noch viel schlimmer - dass das Schweigen ein Verschweigen ist.

Wenn Patienten merken, dass etwas irgendwie schiefläuft, aber niemand es anspricht, vermuten sie erschreckend oft, dass etwas Schlimmes verschwiegen oder gar vertuscht werde. Einsilbige Äußerungen, schnelle Abfertigung und mangelnder Blickkontakt werden dann schnell überbewertet. Das schadet dem Vertrauensverhältnis und führt zu Stress. Dadurch leidet am Ende auch die Compliance, und die unbequeme Gefühlslage verlangsamt womöglich die Heilung.

\section{Entschuldigungen dürfen kurz und direkt sein}

Dabei kostet ein aufrichtiges Wort des Bedauerns oder eine freundliche Entschuldigung kein Geld, erfordert keinen Aufwand und nimmt nicht einmal nennenswert Zeit in Anspruch. Im Gegenteil: Wenn Sie ein Ärgernis mit einer Entschuldigung anerkennen, kann das lange Diskussionen darüber sparen, ob man die Nebenwirkungen oder die Wartezeiten hätte vermeiden können und müssen.

Worte der Entschuldigung oder des Bedauerns müssen keine Elegien sein oder ausschweifende Erklärungen zu Ihrer Entlastung enthalten. Sie dürfen kurz und direkt sein, wenn sie aufrichtig, zugewandt und empathisch vorgetragen werden. Und was sollte man sagen?

\section{Gute Kommunikation verbessert das \\ Arzt-Patienten-Verhältnis}

Studien zeigen, dass Patienten, die die Kommunikation mit ihren Ärzten bemängeln, präzise Vorstellungen davon haben, was eine adäquate Äußerung gewesen wäre. US-amerikanische Mediziner haben 78 Krebspatienten befragt, die angaben, mit ihrer Behandlung unzufrieden zu sein [3]. Sie beklagten entweder Mängel bei der Behandlung (chirurgische Probleme, Infektionen, inadäquate Reaktion auf Komplikationen oder Nebenwirkungen, verspätete Diagnosen), Mängel in der Kommunikation (schlechte Aufklärung, unzureichende Information zur Diagnose, nicht Zuhören, emotionale Kälte) oder beides zusammen. Ob Behandlung und Kommunikation tatsächlich unzureichend oder fehlerhaft waren, wurde nicht nachgeprüft. Ausschlaggebend war die Wahrnehmung der
Patienten, die sich schlecht behandelt fühlten.

Gerade in der Onkologie ist das Vertrauensverhältnis zwischen Arzt und Patient besonders wichtig. Studien belegen, dass schlechte Kommunikation nicht nur das Arzt-Patienten-Verhältnis beeinträchtigt, sondern auch mit schlechteren Behandlungsergebnissen einhergeht.

Krebstherapien sind oft komplex, sie strapazieren die Patienten, beschäftigen verschiedene Ärzte, die toxische Substanzen und strapaziöse Eingriffe anwenden. Die Wahrscheinlichkeit ist hoch, dass unerwünschte Wirkungen, Schwierigkeiten, Informationsdefizite und auch wirkliche Fehler auftreten. Gleichzeitig sind Krebspatienten ernsthaft krank, körperlich und seelisch angegriffen und besonders auf medizinische und auch emotionale Unterstützung ihrer Ärzte angewiesen. In 78 Interviews wollten die Forscher wissen, was sich die unzufriedenen Patienten gewünscht hätten und kamen auf folgende Forderungen:

\section{Seien Sie offen}

Patienten können viel aushalten und haben erstaunlich viel Verständnis für menschliches Versagen, wenn es offen kommuniziert wird. Die befragten Patienten wollten genau Bescheid wissen, was mit ihnen und ihrer Behandlung los ist. Außerdem wünschten sie sich, dass bei Fehlern alle Beteiligten informiert werden, damit so etwas nicht wieder passiert.

Konkret hätten sie gern gehört: „Ich habe einen Fehler gemacht, ich hätte Handschuhe anziehen sollen.“ Oder „Es tut mir leid, dass ich das gesagt habe. Das war falsch. Ich hätte warten sollen, bis wir mehr Informationen haben“. Oder einfach: „Es tut mir leid, ich habe einen Fehler gemacht“. Eine Patientin meinte: „Meine Hausärztin war auch traurig, das passte zu meinen Emotionen. Sie versuchte nicht so zu tun als sei nichts passiert. Das machte für mich einen gewaltigen Unterschied. Sie war einfach ehrlich und authentisch".

\section{Zeigen Sie Bedauern und entschuldigen Sie sich}

Eine Entschuldigung schätzen Patienten höher als Information darüber, was falsch gelaufen ist. Einer der Befragten sagte: „Ich 
weiß nicht, ob es mir geholfen hätte, wenn sie das alles erklären. Was ich wirklich wollte, war, dass sich einer kümmert und dass er sagt: oh, das tut mir so leid“. Eine Patientin gab an, eine Entschuldigung hätte „mir ein besseres Gefühl gegeben mit dem Team und der Behandlung, denn das hätte mir gezeigt, dass sie wirklich wissen, was sie tun und dass sie ihre Patienten verstehen“.

Wenn Patienten eine Entschuldigung oder andere Äußerung des Mitgefühls, der Sorge oder des Bedauerns zu hören bekamen, so lobten sie das ausdrücklich: „Die Tatsache, dass jemand anderes sich für die Aktionen der Ärztin entschuldigte, zeigte mir, dass er mitfühlte und es ihm leid tat, dass mir das passiert ist“. Ein anderer Patient sagte zu einer Entschuldigung: „Ich denke, sie tat das richtige... Sie erkannte an, dass es für mich eine ziemlich schreckliche Erfahrung war".

\section{Übernehmen Sie Verantwortung}

Überzeugend wirkt eine Entschuldigung nur, wenn jemand damit auch Verantwortung übernimmt. Das Wort (responsibility) taucht in fast allen Interviews auf. Für die Patienten ist die Vorstellung furchtbar, dass Ärzten der gleiche Fehler immer wieder passiert. Ein Patient gab an: „Er hätte wenigstens sagen sollen: Mann, das habe ich übersehen. Tut mir leid, dass das passiert ist. Das ist mir noch nie passiert“. Die Befragten möchten, dass die Klinik aus Fehlern lernt und sich so verbessert. Ein Patient sagte: „Es hat mit Verantwortung zu tun. Ich möchte nicht hören ,tut mir leid‘. Tut mir leid heißt gar nichts. Ich möchte sehen, was sie tun, um das Problem zu beheben. Sagt mir nicht, dass es euch leid tut, dass das Problem aufgetreten ist... ich will Resultate sehen“. Ein anderer sagte: „Für mich bedeutet es sehr viel, wenn jemand anerkennt: Ich habe einen Fehler gemacht. Und es bedeutet mir sogar noch mehr, wenn er sagt, wie er das künftig vermeiden will. Aber das habe ich nie gehört. Das habe ich von der Chirurgin nie gehört, und ich weiß wirklich nicht, ob sie überhaupt denkt, dass sie einen Fehler gemacht hat".

Interessanterweise spielte Schadensersatz bei den 78 Interviews keine große Rolle, obwohl es in den USA aufgrund ihrer Rechtsprechung weit üblicher ist auf Schadensersatz zu klagen als bei uns. Nur 2 der Befragten hätten gern die zusätzlichen Kosten erstattet bekommen, die ihnen durch mögliche ärztliche Fehler entstanden waren.

\section{Entschuldigen hilft auch im Team}

Nicht nur zwischen Arzt und Patient, auch unter Kollegen oder im Zusammenspiel mit Pflegekräften, Therapeuten und anderen Professionen wirkt eine Entschuldigung Wunder. Ein so großer und komplexer Betrieb wie eine Klinik bietet täglich tausendfach Anlass, ein Wort des Bedauerns loszuwerden. Wenn Ärzte es gegenüber Pflegenden unterlassen, wird das als arrogant ausgelegt. „Tut mir leid, dass ihr mit der Visite warten musstet" verbessert auch das Klima auf der Station, es zeigt Wertschätzung und verbessert die Atmosphäre und damit die Zusammenarbeit innerhalb und mit anderen Disziplinen.

\section{Zitierweise für diesen Artikel}

klinikarzt 2020; 49: 351-353
Autor

Jutta von Campenhausen

\section{Korrespondenzadresse}

Dr. Jutta von Campenhausen
Institut für Geschichte und Ethik der Medizin
Universitätsklinikum Hamburg - Eppendorf
Martinistraße 52
Gebäude N 30 b
20246 Hamburg
Deutschland
j.von-campenhausen@uke.de

\section{Literatur}

[1] Whited MC, Wheat AL, Larkin KT. The influence of forgiveness and apology on cardiovascular reactivity and recovery in response to mental stress. J Behav Med 2010; 33: 293

[2] Brooks AW, Hengchen D, Schweitzer ME. I'm Sorry About the Rain! Superfluous Apologies Demonstrate Empathic Concern and Increase Trust. Social Psychological and Personality. Science 2014; 5: 467-474

[3] Mazor KM, Greene SM, Roblin D et al. More Than Words: Patients' Views on Apology and Disclosure When Things Go Wrong in Cancer Care. Patient Educ Couns 2013; 90 : 341-346

Bibliografie

Akt Rheumatol 2022; 47: 25-27

DOI $10.1055 / \mathrm{a}-1445-9872$

ISSN 0034-3536

(c) 2022. Thieme. All rights reserved. Georg Thieme Verlag KG, Rüdigerstraße 14, 70469 Stuttgart, Germany 